\title{
ETIOLOGIAS DA DISFUNÇÃO SISTÓLICA EM CÃES
}

\author{
COSTA, Suyan Andrade ${ }^{1}$; \\ SOARES, Frederico Aécio Carvalho ${ }^{2}$.
}

Recebido: 06/08/2019

Aceito: $27 / 11 / 2019$

${ }^{1}$ Acadêmica, Faculdade de Medicina Veterinária, Centro Universitário Ritter dos Reis - UniRitter; ${ }^{2}$ Professor Auxiliar, Faculdade de Medicina Veterinária, Centro Universitário Ritter dos Reis - UniRitter.

\section{RESUMO}

\begin{abstract}
A
Iterações cardíacas podem causar grande impacto hemodinâmico nos cães. Quando as funções do coração estão reduzidas, o organismo ativa mecanismos compensatórios que podem levar a insuficiência cardíaca. A disfunção sistólica é a perda da capacidade de contratilidade do coração, com consequente insuficiência miocárdica, que pode ser causada por sobrecarga de volume, sobrecarga de pressão e/ou disfunção miocárdica. Inúmeras patologias e etiologias podem cursar com disfunção sistólica, sendo as principais: cardiomiopatia dilatada, degeneração valvar em estágios avançados, hipotireoidismo, endocardite, miocardite, deficiência nutricional de taurina, taquiarritmias persistentes e toxicidade por doxorrubicina. A disfunção sistólica é detectada através do exame de ecocardiografia, através de parâmetros como a fração de ejeção ventricular, a fração de encurtamento e a distância E-septo. O tratamento da disfunção sistólica depende da causa e dos sinais clínicos envolvidos. Os principais fármacos utilizados no tratamento são os inotrópicos positivos, como digitálicos, simpaticomiméticos e inibidores das fosfodiesterases. O objetivo do presente trabalho foi revisar as principais causas da disfunção sistólica em cães, abordando a sua fisiopatologia, bem como o seu diagnóstico e a sua abordagem terapêutica.
\end{abstract}

Palavras-chave: Insuficiência miocárdica. Contratilidade cardíaca. Disfunção ventricular. 


\section{INTRODUÇÃO}

A cardiologia possui uma grande importância na clínica de pequenos animais, uma vez que é estimada a ocorrência de doenças cardíacas em 10\% dos cães atendidos nas clínicas veterinárias (KEENE et al., 2019). Tais doenças podem ser de origem congênita ou adquirida. Nos cães com idade mais avançada, a prevalência de doenças cardíacas atinge mais de $60 \%$ (ATKINS et al., 2009). Um estudo realizado por Castro et al. (2009) mostrou que, de 854 cães avaliados através de ecocardiografia no Hospital Veterinário da Universidade Federal de Minas Gerais, $750(87,8 \%)$ apresentaram alterações cardíacas. As alterações podem estar relacionadas com disfunções mecânicas ou elétricas, sendo que as disfunções mecânicas compreendem a disfunção diastólica (relaxamento) e a disfunção sistólica (contratilidade). Diversas patologias causam disfunção na contratilidade miocárdica, acarretando sinais clínicos específicos em consequência do baixo débito cardíaco e da insuficiência cardíaca congestiva (CORNELL et al., 2004; HÄGGSTRÖM et al., 2010). Geralmente, as patologias que cursam com disfunção sistólica têm um prognóstico reservado (MOONARMART et al., 2010; OLSEN et al., 2010). Assim, é importante para o médico veterinário de pequenos animais, conhecer a fisiologia cardíaca e os fatores que podem prejudicar a contratilidade miocárdica, visando uma maior precisão diagnóstica e uma melhor abordagem terapêutica. O objetivo do presente trabalho foi revisar as principais causas da disfunção sistólica em cães, abordando sua fisiopatologia, bem como o seu diagnóstico e a sua abordagem terapêutica.

\section{DISFUNÇÃO SISTÓLICA}

Uma das principais funções do coração é contrair para ejetar o sangue necessário para as circulações pulmonar e sistêmica. O volume de sangue ejetado por minuto é chamado de débito cardíaco e pode ser influenciado pela frequência cardíaca, contratilidade do miocárdio, pré-carga (volume de sangue no ventrículo no final da diástole) e pós-carga (resistência a ejeção ventricular, exercida pela resistência vascular sistêmica) (ATKINS et al., 2009). A força de contração do coração para que ocorra a ejeção do sangue e o grau de constrição ou dilatação dos vasos sanguíneos são determinados pelo sistema nervoso autônomo e pelos hormônios produzidos pelos sistemas autócrinos, parácrinos e endócrinos (CUNNINGHAM, 2019). Quando o coração apresenta capacidade de contratilidade 
diminuída, há uma disfunção sistólica, que pode ser resultante de sobrecarga de volume ou de pressão, ou ainda, por doenças que afetam o músculo cardíaco. Visando compensar a disfunção sistólica, o coração sofre modificações adaptativas para manter o equilíbrio circulatório, aumentando o diâmetro e a espessura do ventrículo. As doenças e etiologias mais frequentes relacionadas a insuficiência miocárdica são a cardiomiopatia dilatada, a miocardite e a toxicidade por doxorrubicina (ATKINS et al., 2009; CALABRESE; THIENE, 2003; DEFRANCESCO; HAUCK, 2000).

Na sobrecarga de pressão, o ventrículo encontra resistência para ejetar o volume sanguíneo. Com isso, ocorre uma hipertrofia concêntrica secundária à sobrecarga de pressão e, posteriormente, ocorre uma dilatação da cavidade ventricular para aumentar a contração. Essa hipertrofia concêntrica causa uma fibrose perimuscular, causando prejuízos à função sistólica. As doenças que cursam com disfunção sistólica por sobrecarga de pressão são: a estenose subaórtica, a estenose pulmonar, a hipertensão arterial sistêmica e a hipertensão arterial pulmonar primária ou secundária. Quando ocorre sobrecarga de volume, as câmaras cardíacas aumentam, causando uma hipertrofia excêntrica (ATKINS et al., 2009). Esse aumento de diâmetro das câmaras cardíacas é explicado pelo mecanismo de Frank-Starling, no qual o coração se adapta ao volume de sangue excedente e aumenta sua força de contração em decorrência da sua distensibilidade. Com a progressão da hipertrofia excêntrica, ocorre fibrose no miocárdio, levando a uma disfunção sistólica. As doenças que cursam com sobrecarga de volume são: a degeneração mixomatosa valvar, a endocardite infecciosa, a persistência de ducto arterioso e o defeito de septo ventricular (BOON, 2011; MORAES, 2014).

\section{SINAIS CLÍNICOS DA DISFUNÇÃO SISTÓLICA}

Os sinais clínicos apresentados na disfunção sistólica são resultado da insuficiência cardíaca, que causa acúmulo de fluido, baixo débito cardíaco e alterações no músculo esquelético (MORAES; SCHWARTZ, 2005). Os animais que apresentam disfunção sistólica geralmente demonstram intolerância ao exercício, fraqueza, síncopes e, em graus mais avançados, arritmias cardíacas e morte súbita (CORNELL et al., 2004). O baixo débito cardíaco também compromete a perfusão dos órgãos e tecidos, podendo causar cianose (FILIPPATOS; 
ZANNAD, 2007). Como a disfunção sistólica pode ter diversas patologias e etiologias envolvidas, outros sinais clínicos como tosse, dispneia e perda de peso podem também estar associados (HÄGGSTRÖM et al., 2010).

\section{DIAGNÓSTICO}

A ecocardiografia é o método de escolha e o mais utilizado para investigação de disfunção sistólica em cães (BONAGURA; SCHOBER, 2009; CHETBOUL et al., 2016). Os parâmetros mais utilizados para a avaliação da função sistólica são a fração de ejeção, a fração de encurtamento e a distância E-septo, avaliados através de ecocardiografia (Figura 1) em modo unidimensional (modo M) e bidimensional. O eletrocardiograma não avalia a função sistólica, no entanto, é o exame padrão-ouro para avaliação da função elétrica do coração e é de extrema importância para diagnosticar as alterações de ritmo que cursam com insuficiência miocárdica (BONAGURA; SCHOBER, 2009; FILIPPI, 2011).

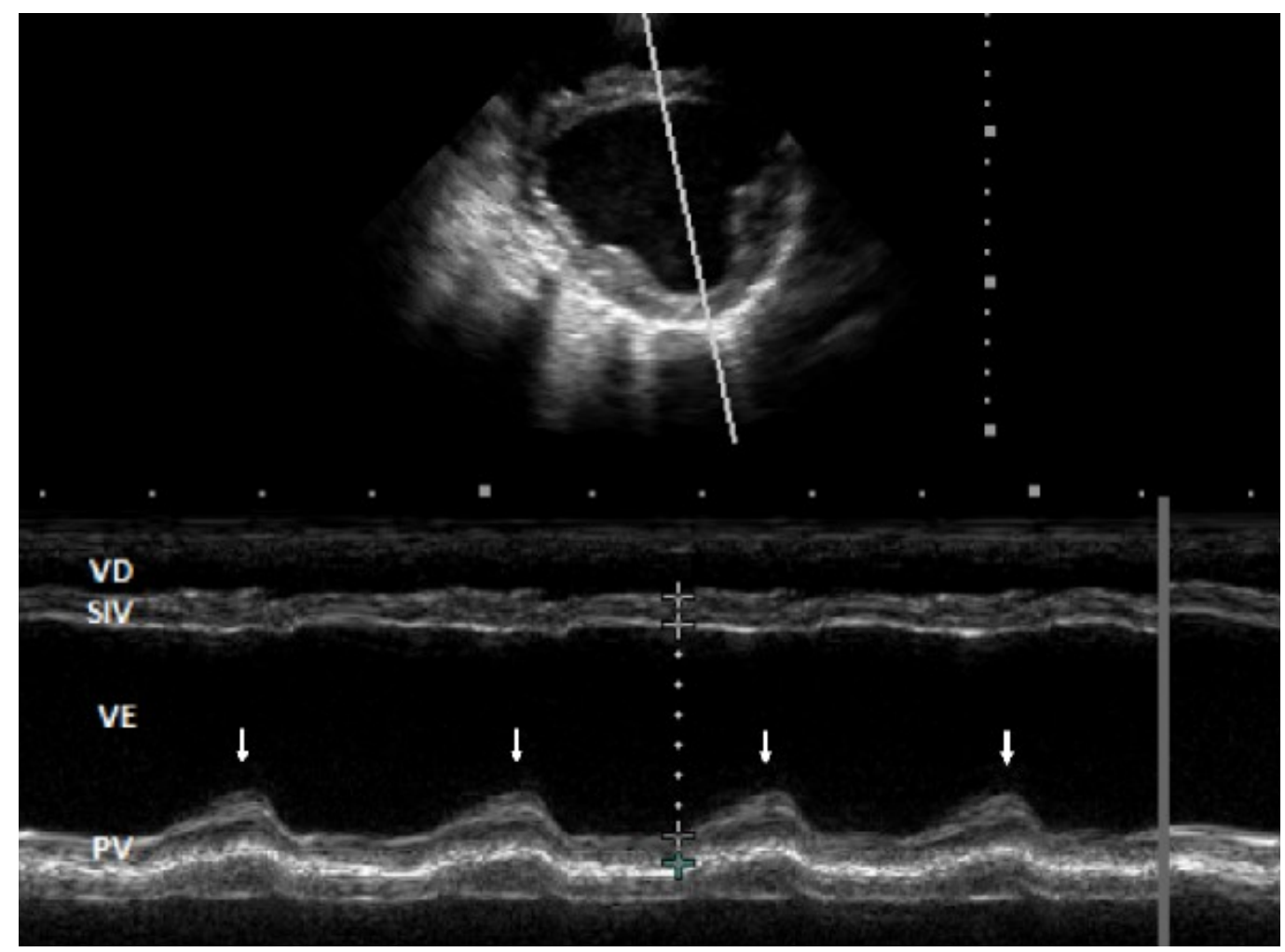

Figura 1 - Imagem ecocardiográfica em modo unidimensional (modo M) para avaliação da função sistólica em paciente canino com disfunção sistólica. VD, cavidade do ventrículo direito em diástole; SIV, septo interventricular em diástole; VE, cavidade do ventrículo esquerdo em diástole; PV, parede do ventrículo esquerdo em diástole. Fonte: Arquivo pessoal dos autores. 


\section{Fração de Ejeção (FE)}

O percentual de sangue ejetado pelo ventrículo esquerdo através da aorta na sístole é representado pela FE. É calculado através de uma diferença entre o volume diastólico e o volume sistólico. Existem diversos métodos para o cálculo da fração de ejeção. Porém, o coração é uma estrutura tridimensional e seu volume é mensurado por um método bidimensional. Logo, o resultado será aproximado. O método mais utilizado é o método de Teicholz, conhecido como método geométrico. Porém esse método possui limitações conforme a cavidade do ventrículo esquerdo (VE) aumenta de tamanho. É utilizado um intervalo de referência que pode variar de acordo com cada autor. Este intervalo varia de 60 a 79,6\% para cães (BOON, 2011; CHETBOUL et al., 2016). Ocorre alteração na FE quando o volume do ventrículo esquerdo é alterado entre a sístole e a diástole. O método de Simpson modificado, também conhecido como modelo dos discos, oferece boa acurácia pois independe da geometria ventricular para obter o volume calculado (BONAGURA; SCHOBER, 2009; BOON, 2011).

\section{Fração de Encurtamento (FS)}

A fração de encurtamento é um índice obtido a partir do modo $M$ do VE, através da janela paraesternal direita, calculado utilizando as variações das dimensões internas do VE em sístole e diástole (SOUSA; CARVALHO, 2019). Os fatores envolvidos na FS são a pré-carga, a pós-carga e a contratilidade. Este método depende de vários fatores, como a qualidade da imagem e a experiência do examinador (PIVETA et al., 2012). O aumento na pré-carga, a diminuição da pós-carga ou o aumento da contratilidade aumentam o valor da FS. Já a diminuição na pré-carga ou o aumento na pós-carga, diminuem o valor da FS. Um valor baixo de FS sugere diminuição da contratilidade. Em cães, esse índice varia conforme o porte e a raça, mas é utilizado um intervalo de referência que pode variar de acordo com cada autor. Este intervalo varia de 29 a 50\% para cães de pequeno a médio porte e de 25 a 50\% para cães de grande porte (BONAGURA; SCHOBER, 2009; BULWER et al., 2007; SOUSA; CARVALHO, 2019). 


\section{Distância E-septo}

Mensurado através do modo $\mathrm{M}$, é a distância do ponto $\mathrm{E}$ do folheto anterior da válvula mitral ao septo interventricular, no seu limite inferior. Este é um parâmetro ecocardiográfico de avaliação do preenchimento e função ventriculares (HOLLER; WESS, 2014). Nas patologias onde há sobrecarga de volume com disfunção sistólica, a distância do folheto da mitral ao septo aumenta. Estudos em pacientes caninos com cardiomiopatia dilatada evidenciaram altas sensibilidade e especificidade da distância E-septo para identificar a disfunção sistólica e auxiliar no diagnóstico da doença (HOLLER; WESS, 2014; VOLLMAR, 1999). O valor de referência de normalidade utilizado para a distância E-septo é de até 8 mm (BOON, 2011; WARE, 2011).

\section{Outros métodos de avaliação da função sistólica}

Além dos parâmetros já citados anteriormente, outros índices podem ser utilizados para avaliar a função sistólica, como o diâmetro sistólico final, o volume sistólico final, o volume sistólico final indexado à área de superfície corporal, a porcentagem de aumento do diâmetro interno do ventrículo esquerdo na sístole, a taxa de aumento de pressão do VE $(+\mathrm{dP} / \mathrm{dt})$, a movimentação anular mitral (MAM) e a movimentação anular tricúspide (TAPSE) (CHETBOUL; TISSIER, 2012; PASLAWSKA et al., 2012).

Com a necessidade de se obter informações mais precisas na avaliação da contratilidade cardíaca, novas técnicas e tecnologias estão sendo utilizadas na medicina veterinária, como o Doppler Tecidual (avalia a velocidade de movimentação do miocárdio), a técnica do Speckle Tracking (avalia percentual de deformidade miocárdica), a ecocardiografia tridimensional em tempo real, a ressonância magnética e a tomografia computadorizada. Essas técnicas e exames de imagem permitem obter um diagnóstico mais precoce da disfunção sistólica (ESKOFIER et al., 2015; SIESLACK et al., 2013; WOLF et al., 2018). 


\section{PRINCIPAIS PATOLOGIAS E ETIOLOGIAS QUE CURSAM COM DISFUNÇÃO SISTÓLICA}

\section{Cardiomiopatia dilatada}

A cardiomiopatia dilatada é a doença miocárdica adquirida, mais frequente em cães de raças grandes e gigantes, mas pode ocorrer também em raças de pequeno e médio porte. Ocorre perda da função sistólica e hipertrofia excêntrica das câmaras cardíacas (MARTINS, 2017). Muitas vezes, os pacientes podem desenvolver arritmias, com maior prevalência de fibrilação atrial. A principal causa da cardiomiopatia dilatada é primária, de origem genética. Os cães da raça Dobermann são os mais atingidos pela doença. A doença na raça é hereditária e ocorre por transmissão de gene autossômico dominante, alterando genes codificadores de proteínas contráteis (WESS et al., 2017). Estudos realizados no Canadá e na Europa detectaram alta prevalência da doença na raça, com resultados de $63 \%$ e $58 \%$, respectivamente (CALVERT; MEURS, 2000; WESS et al., 2010). Exames de rotina são de extrema importância, uma vez que a doença pode estar oculta (SISSON et al., 2014). O diagnóstico da cardiomiopatia dilatada é realizado por exclusão, visto que outras doenças podem causar alterações ecocardiográficas similares. Um estudo revelou que de 13 cães diagnosticados com cardiomiopatia dilatada, 12 apresentaram, ao exame ecocardiográfico, redução da fração de encurtamento (MURTA, 2009).

\section{Degeneração mixomatosa valvar}

A degeneração mixomatosa valvar é a doença cardíaca mais comum na medicina veterinária. É uma doença crônica e degenerativa, com caráter hereditário, que atinge cães de pequeno porte, geralmente a partir dos oito anos de idade (DILLON et al., 2012; KEENE et al., 2019). Ocorre com maior frequência na valva mitral, podendo afetar também a valva tricúspide. A degeneração progressiva da válvula impede sua correta coaptação, permitindo que parte do sangue retorne ao átrio. Essa regurgitação progressiva leva ao remodelamento cardíaco (KEENE et al., 2019). Mecanismos compensatórios são ativados - sistema reninaangiotensina-aldosterona e aumento da atividade simpática - para suprir as necessidades circulatórias. Com o aumento das dimensões do ventrículo esquerdo, em casos mais avançados, há diminuição na função sistólica (FRUTUOSO, 2015; HÄGGSTRÖM et al., 2010). Um estudo realizado por Borgarelli et al. (2007) mostrou que todos os 56 cães da pesquisa, 
em estágios mais avançados da degeneração valvar, apresentaram disfunção sistólica, verificada através do exame de ecocardiografia.

\section{Endocardite}

A endocardite é uma infecção bacteriana do endocárdio que pode acometer valvas atrioventriculares e semilunares, a partir de uma porta de entrada para infecção e colonização. Essas alterações causam uma insuficiência valvular, com consequente regurgitação e sobrecarga de volume (BOON, 2011; WARE, 2011). A disfunção sistólica do ventrículo esquerdo ocorre por decorrência do processo infeccioso agudo nas valvas, que impede a correta coaptação dos folhetos valvares e cursa com sobrecarga de volume. $\mathrm{O}$ exame de ecocardiografia mostra o comprometimento cardíaco por consequência da deformidade das valvas e insuficiência cardíaca (ABBOT, 2015). A disfunção sistólica já foi relatada em um paciente canino com endocardite infecciosa da valva aórtica associada à persistência de ducto arterioso, uma cardiopatia congênita (AOKI et al., 2015).

\section{Hipotireoidismo}

O hipotireoidismo é uma endocrinopatia que costuma acometer cães de médio a grande porte, entre quatro e oito anos de idade, podendo ser classificado como primário, quando há destruição da glândula tireóide, ou central, quando há doença hipotalâmica ou hipofisária (VOORBIJ et al., 2016). A deficiência de hormônios tireoidianos compromete a função de diversos sistemas corporais, inclusive o cardiovascular. A doença altera a expressão gênica cardíaca, pois o hormônio triiodotironina (T3) influi na expressão de genes cardíacos específicos que envolvem a contratilidade. O hormônio T3 está diretamente relacionado à função contrátil do coração. Baixas concentrações dos hormônios tireoidianos levam a uma redução na contratilidade e frequência cardíaca, causando uma redução do débito cardíaco. Logo, o impacto cardiovascular é evidenciado através de bradicardia, diminuição dos efeitos cronotrópicos (frequência) e inotrópicos (contratilidade), além de arritmias (CHASTAIN, 1999; CRUZ; MANOEL, 2015; FELDMAN; NELSON, 2004). Em um estudo realizado por Beier et al. (2015) que incluiu 23 cães com diagnóstico de hipotireoidismo, 12 animais apresentaram alterações ecocardiográficas compatíveis com disfunção sistólica. 


\section{Miocardite}

A miocardite é uma afecção decorrente de inflamação no músculo cardíaco e pode ser causada por agentes infecciosos ou não-infecciosos. A inflamação causa lesões nos cardiomiócitos, levando à degeneração do músculo e cardiomegalia. Estas lesões interferem na contratilidade do miocárdio, assim, o coração perde a capacidade de bombear a quantidade adequada de sangue, apresentando baixo débito cardíaco e insuficiência cardíaca. Em cães, a doença pode ocorrer em decorrência de infecções por parvovirose ou leishmaniose, além de casos de septicemia e doença de Lyme (ELAMM et al., 2012). A miocardite pode causar arritmias cardíacas persistentes e prejudicar a contratilidade cardíaca. No caso da parvovirose, pode resultar em cardiomiopatia dilatada em cães jovens que sobreviveram à infecção. O herpesvírus em cães filhotes também pode causar miocardite necrosante (WARE, 2011). A miocardite pode ocorrer também em consequência da doença de Chagas, que é transmitida por insetos vetores da família Reduviidae, conhecidos popularmente por barbeiros. O protozoário causador da doença é o Trypanosoma cruzi. Tanto homens quanto cães podem ter manifestações cardíacas causadas por essa zoonose. A doença é conhecida por causar, em sua fase crônica sintomática, uma cardiomiopatia chagásica, que afeta a contratilidade e o relaxamento miocárdico e é caracterizada por cardiomegalia e alterações no ritmo devido a cardiomiopatia inflamatória fibrosante, verificados através dos exames ecocardiográfico e eletrocardiográfico (CUNHA, 2017). Em um estudo realizado no estado do Mato Grosso do Sul por Souza et al. (2008), os cães positivos para doença de Chagas, submetidos ao exame ecocardiográfico, apresentaram redução na fração de encurtamento, sugestiva de disfunção sistólica.

\section{Deficiência nutricional}

Entre os aminoácidos livres do coração, a taurina é o que está em maior quantidade. A deficiência dos substratos metabólicos L-carnitina ou taurina em cães foi identificada como possível causadora de cardiomiopatia dilatada por deficiência nutricional. A carnitina transporta aminoácidos livres de cadeia longa para dentro das mitocôndrias. Com doses baixas desse nutriente, o coração fica sem o combustível metabólico adequado para desempenhar corretamente a sua função (O'GRADY; O'SULLIVAN, 2004). Um estudo 
experimental com ratos evidenciou que a administração de L-carnitina em condições de hipóxia melhora significativamente a contratilidade dos cardiomiócitos (XUE et al., 2017). Em um estudo realizado por Sisson et al. (1999), cães com cardiomiopatia dilatada apresentaram níveis baixos de taurina, porém, quando suplementados com o aminoácido, suas funções miocárdicas apresentaram melhora. Outro estudo, que avaliou 24 cães da raça Golden Retriever com diagnóstico de deficiência de taurina e cardiomiopatia dilatada, evidenciou que todos os cães apresentaram FS menores que $25 \%$, e 23 destes cães estavam sendo alimentados com dietas livres de grãos, ricas em leguminosas ou a combinação dos dois (KAPLAN et al., 2018). Ainda, um recente estudo retrospectivo associou o fenótipo cardiomiopatia dilatada com certas dietas livres de grãos (ADIN et al., 2019).

\section{Taquiarritmias persistentes}

Os distúrbios de ritmo podem ser classificados em distúrbios de condução, formação ou distúrbios complexos, que envolvem condução e formação do impulso elétrico cardíaco. A excitabilidade aumentada persistente causa taquiarritmias nos cães (MILLER et al., 1999). Períodos crônicos de taquiarritmias podem promover uma ineficiente contratilidade do coração, prejudicando o bombeamento do sangue, a perfusão do miocárdio e o débito cardíaco, devido à exaustão dos mecanismos compensatórios, o que leva à dilatação e hipertrofia do miocárdio. Sendo assim, as arritmias supraventriculares e ventriculares são prejudiciais ao correto funcionamento cardíaco. Como exemplo de taquiarritmias persistentes, estão a taquicardia atrial sustentada, a taquicardia ventricular sustentada e a fibrilação atrial (FILIPPI, 2011; SANTILLI; PEREGO, 2009). Estas arritmias possuem gravidades diferentes. A fibrilação atrial (Figura 2) é uma arritmia bastante comum em casos de aumento atrial importante e que pode ser controlada com a utilização de fármacos antiarrítmicos. Já a taquicardia ventricular sustentada é considerada uma arritmia grave, de tratamento imediato, pois o coração não bombeia adequadamente o sangue para a circulação (SANTILLI; PEREGO, 2009). O tratamento das arritmias ventriculares pode ser feito com fármacos antiarrítmicos classe III ou beta-bloqueadores. Porém, esses fármacos devem ser utilizados com cautela, já que possuem efeito de redução da função sistólica (SPINOSA et al., 2006). 


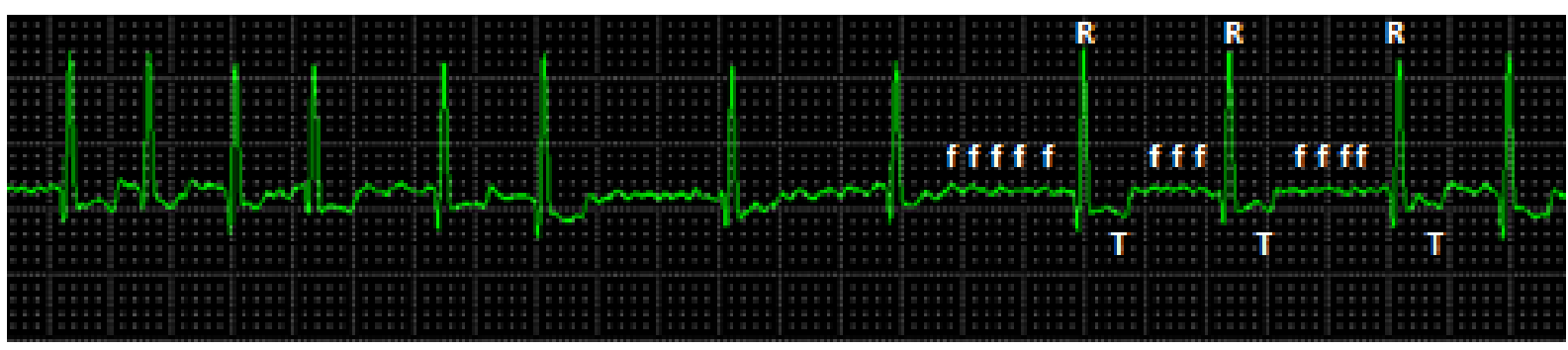

Figura 2 - Imagem eletrocardiográfica (derivação II) de um paciente canino com fibrilação atrial. R: ondas R; T: ondas T; f: ondas f. Fonte: Arquivo pessoal dos autores.

\section{Toxicidade por doxorrubicina}

A maioria dos quimioterápicos causam toxicidade nas células do organismo, ou por tempo de uso do fármaco ou por concentração. Dentre eles, alguns causam maiores danos nas células cardíacas (VIÉRA, 2015). A doxorrubicina é um fármaco utilizado com bastante frequência em oncologia veterinária, principalmente nos tratamentos de tumores malignos sólidos e hematopoiéticos de diferentes origens, como o linfoma, o hemangiossarcoma e o osteossarcoma (DANMAIGORO et al., 2018). Pertencente a um grupo de antibióticos antitumorais, é o fármaco com maior ocorrência de cardiotoxicidade por acúmulo de doses. Esta cardiotoxicidade, causada principalmente pelo estresse oxidativo, pode se tornar crônica, levando a uma disfunção sistólica irreversível e refratária aos tratamentos adjuvantes da contratilidade cardíaca (OCTAVIA et al., 2012). A dose utilizada em medicina veterinária, mantendo uma margem de segurança, não deve ultrapassar o intervalo de 120 a $150 \mathrm{mg} / \mathrm{m} 2$, o que equivale de quatro a cinco administrações de doxorrubicina (GUSTAFSON; PAGE, 2013). Em cães, as doses sugeridas em cada aplicação são: 20 mg/m2 (dose baixa), 30 $\mathrm{mg} / \mathrm{m} 2$ (dose média) e $50 \mathrm{mg} / \mathrm{m} 2$ (dose alta) (DANMAIGORO et al., 2018). Cães que utilizaram as doses altas nem sempre apresentaram toxicidade, enquanto cães submetidos ao tratamento com doses baixas podem apresentar toxicidade cardíaca já nas primeiras doses. As alterações causadas pela doxorrubicina no coração são semelhantes à cardiomiopatia dilatada, com aumento das câmaras cardíacas e disfunção sistólica. Além disso, a doxorrubicina pode afetar a concentração de cálcio intracelular, reduzindo a contratilidade cardíaca (BOON, 2011). Em um estudo realizado por Viéra (2015), de dez cadelas tratadas com doxorrubicina para tumor venéreo transmissível, duas apresentaram 
redução da fração de encurtamento (abaixo de $25 \%$ ) antes de terminarem o protocolo prescrito. Uma das pacientes recebeu dose cumulativa de $60 \mathrm{mg} / \mathrm{m} 2$ (duas aplicações) e outra de $90 \mathrm{mg} / \mathrm{m} 2$ (três aplicações). A realização de ecocardiografia e eletrocardiografia periódica nos pacientes em tratamento é fundamental, uma vez que a redução da fração de encurtamento e a presença de batimentos prematuros podem ser indícios de cardiotoxicidade (HALLMAN et al., 2019).

\section{TRATAMENTO DA DISFUNÇÃO SISTÓLICA}

Geralmente, o tratamento da disfunção sistólica é realizado com o uso de fármacos inotrópicos positivos, que aumentam a contratilidade cardíaca e o volume de ejeção. Agem nos receptores beta-adrenérgicos, localizados no miocárdio, terminações adrenérgicas e musculatura lisa de vasos. As principais classes de inotrópicos positivos utilizados na medicina veterinária são os digitálicos, simpaticomiméticos e inibidores das fosfodiesterases. A escolha dos fármacos, suas doses e associações, dependerá dos sinais clínicos do paciente e do ambiente no qual ele será tratado, domiciliar ou hospitalar (VIDAL, 2016). Além do tratamento da disfunção sistólica em si, é fundamental o tratamento de sua causa de base, bem como o tratamento da insuficiência cardíaca com outras classes de fármacos, como diuréticos e vasodilatadores (KEENE et al., 2019; TILLEY et al., 2015).

\section{Digitálicos}

Os fármacos glicosídeos digitálicos, como a digoxina, alteram o tônus simpático, diminuindo a condução do impulso elétrico através dos átrios e do nodo atrioventricular. Agem inibindo as enzimas adenosinas trifosfatases, aumentado as concentrações de cálcio intracelular dos miócitos e, consequentemente, aumentando a contração cardíaca (WASSERSTROM; AISTRUP, 2005). A digoxina é o principal digitálico indicado para pacientes com insuficiência cardíaca, disfunção sistólica e fibrilação atrial, possuindo atividade direta na contratilidade cardíaca (RAHIMTOOLA, 2004). A dose recomendada para cães é de 0,0025 a 0,005 mg/kg, administrada por via oral, a cada 12 horas (KEENE et al., 2019). 


\section{Simpaticomiméticos}

Os fármacos simpaticomiméticos são representados principalmente pela dopamina e pela dobutamina, aumentam o volume sistólico e o débito cardíaco. A dobutamina é bastante utilizada em ambiente hospitalar, na ressuscitação do choque séptico em cães, para melhorar o débito cardíaco e corrigir a hipoperfusão. Possuem ação direta, ligando-se aos receptores adrenérgicos, simulando os efeitos das catecolaminas: adrenalina e noradrenalina (RHODES et al., 2017). A dose recomendada de dobutamina para cães é de 2,5 a $10 \mathrm{\mu g} / \mathrm{kg} / \mathrm{min}$, em infusão contínua (KEENE et al., 2019).

\section{Inibidores das fosfodiesterases}

Os fármacos inotrópicos positivos inibidores da fosfodiesterase permitem uma maior entrada de cálcio intracelular, reduzindo a pós-carga, sem provocar alterações no ritmo cardíaco. O pimobendan, inibidor da fosfodiesterase III, possui efeito inotrópico positivo, diminui a resistência vascular sistêmica e a pressão atrial esquerda, e reduz os níveis de citocinas inflamatórias (HOGAN; GREEN, 2009; HORI et al., 2019). A dose recomendada para cães é de 0,25 a 0,3 mg/kg, administrado por via oral, a cada 12 horas (KEENE et al., 2019).

\section{CONCLUSÃO}

O diagnóstico da disfunção sistólica é complexo e envolve o conhecimento de suas diversas etiologias, assim como sua fisiopatologia e sinais clínicos. Para isso, é fundamental a realização de uma anamnese detalhada e de um exame físico completo. Estes dados, aliados ao exame de ecocardiografia, para avaliação da contratilidade cardíaca, são de grande importância para o diagnóstico da disfunção sistólica em cães. E, para que a abordagem terapêutica seja feita de forma correta e eficiente, é necessário o conhecimento da causa de base das alterações cardíacas. 


\section{ETIOLOGIES OF SYSTOLIC DYSFUNCTION IN DOGS}

\section{ABSTRACT}

C ardiac changes can cause a major hemodynamic impact in dogs. When heart functions are reduced, the body activates compensatory mechanisms that can lead to heart failure. Systolic dysfunction is the loss of heart contractility capacity, with consequent myocardial insufficiency that can be caused by volume overload, pressure overload and/or myocardial dysfunction. Numerous pathologies and etiologies may present with systolic dysfunction, the main ones being: dilated cardiomyopathy, valve degeneration in advanced stages, hypothyroidism, endocarditis, myocarditis, nutritional taurine deficiency, persistent tachyarrhythmias, and doxorubicin toxicity. Systolic dysfunction is detected by echocardiography, through parameters such as ventricular ejection fraction, shortening fraction, and E-septum distance. The treatment of systolic dysfunction depends on the cause and clinical signs involved. The main drugs used in the treatment are positive inotropic drugs, such as digitalis, sympathomimetics, and phosphodiesterase inhibitors. The aim of the present study was to review the main causes of systolic dysfunction in dogs, addressing their pathophysiology, as well as its diagnosis and therapeutic approach.

Keywords: Myocardial insufficiency. Cardiac contractility. Left ventricular dysfunction.

\section{ETIOLOGIAS DE LA DISFUNCIÓN SISTÓLICA EN PERROS}

\section{RESUMEN}

os cambios cardíacos pueden causar un impacto hemodinámico importante en los perros. Cuando se reducen las funciones del corazón, el cuerpo activa los mecanismos compensatorios que pueden conducir a la insuficiencia cardíaca. La disfunción sistólica es la pérdida de la capacidad de contractilidad del corazón, con la consiguiente insuficiencia miocárdica que puede ser causada por sobrecarga de volumen, sobrecarga de presión y/o disfunción miocárdica. Numerosas patologías y etiologías pueden presentar disfunción sistólica, siendo las principales: cardiomiopatía dilatada, degeneración valvular avanzada, hipotiroidismo, endocarditis, miocarditis, deficiencia nutricional de taurina, taquiarritmias persistentes y toxicidad por doxorrubicina. La disfunción sistólica se detecta por ecocardiografía, a través de parámetros como la fracción de eyección ventricular, la fracción de acortamiento y la distancia E-septum. El tratamiento de la disfunción sistólica depende de la causa y los signos clínicos involucrados. Los principales fármacos utilizados en el tratamiento son los inotrópicos positivos, como digitálicos, simpaticomiméticos y inhibidores de las fosfodiesterasas. El objetivo del presente estudio fue revisar las principales causas de la disfunción sistólica en perros, abordando su fisiopatología, así como su diagnóstico y su enfoque terapéutico.

Palabras clave: Insuficiencia miocárdica. Contratilidad cardiaca. Disfunción ventricular izquierda. 


\section{REFERÊNCIAS}

ABBOT, J. A. Acquired Valvular Disease. In: TILLEY, L. P.; SMITH, F. W.; OYAMA, M.; et al. Manual of Canine and Feline Cardiology. St. Louis: Elsevier Saunders, 2015. P. 133-140.

ADIN, D.; DEFRANCESCO, T. C.; KEENE, B.; et al. Echocardiographic phenotype of canine dilated cardiomyopathy differs based on diet type. Journal of Veterinary Cardiology, v. 21, p. 1-9, 2019.

AOKI, T.; SUNAHARA, H.; SUGIMOTO, K.; et al. Infective endocarditis of the aortic valve in a Border collie dog with patent ductus arteriosus. Journal of Veterinary Medical Science, $v$. 77, n. 3, p. 331-336, 2015.

ATKINS, C.; BONAGURA, J.; ETTINGER, S.; et al. Guidelines for the diagnosis and treatment of canine chronic valvular heart disease. Journal of Veterinary Internal Medicine, v. 77, n. 3, p. 1142-1150, 2009.

BEIER, P.; REESE, S.; HOLLER, P. J.; et al. The Role of Hypothyroidism in the Etiology and Progression of Dilated Cardiomyopathy in Doberman Pinschers. Journal of Veterinary Internal Medicine, v. 29, n. 1, p. 141-149, 2015.

BONAGURA, J. D.; SCHOBER, K. E. Can ventricular function be assessed by echocardiography in chronic canine mitral valve disease? The Journal of Small Animal Practice, v. 50, n. 1, p. 12-24, 2009.

BOON, J. A. Veterinary Echocardiography. 2. ed. Wiley-Blackwell, 2011. 632p.

BORGARELLI, M.; TARDUCCI, A.; ZANATTA, R.; et al. Decreased systolic function and inadequate hypertrophy in large and small breed dogs with chronic mitral valve insufficiency. Journal of Veterinary Internal Medicine, v. 21, n. 1, p. 61-67, 2007.

BULWER, B.; SOLOMON, S.; JANARDHANANJ, R. Echocardiographic Assessment of Ventricular Systolic Function. In: SOLOMON, S. Essential Echocardiography. New Jersey: Humana Press. 2007. P. 98-99.

CALABRESE, F.; THIENE, G. Myocarditis and inflammatory cardiomyopathy: microbiological and molecular biological aspects. Cardiovascular Research, v. 60, n. 1, p. 11-25, 2003.

CALVERT, C. A.; MEURS, K. CVT update: Doberman Pinscher occult cardiomyopathy. In: BONAGURA, J. D.; KIRK, R. W. Kirk's Current Veterinary Therapy. Philadelphia: Saunders. 2000. P. 756-760. 
CASTRO, M. G.; VEADO, J. C. C.; SILVA, E. F.; et al. Estudo retrospectivo ecodopplercardiográfico das principais cardiopatias diagnosticadas em cães. Arquivo Brasileiro de Medicina Veterinária e Zootecnia, v. 61, n. 5, p. 1238-1241, 2009.

CHASTAIN, C. B. O sistema endócrino e metabólico. In: GOLDSTON, R. T.; HOSKINS, J. D. Geriatria e gerontologia cão e gato. São Paulo: Roca, 1999. 335p.

CHETBOUL, V.; BUSSADORI, C.; MADRON, E. Clinical echocardiography of the dog and cat. 2. ed. Missouri: Elsevier, 2016.

CHETBOUL, V.; TISSIER, R. Echocardiographic assessment of canine degenerative mitral valve disease. Journal of Veterinary Cardiology, v. 14, n. 1, p. 127-148, 2012.

CORNELL, C. C.; KITTLESON, M. D.; DELLA TORRE, P.; et al. Allometric scaling of M-mode cardiac measurements in normal adult dogs. Journal of Veterinary Internal Medicine, v. 18, n. 3, p. 311-321, 2004.

CRUZ, F. G. B.; MANOEL, F. M. T. Hipotireoidismo Canino In: JERICO, M. M.; NETO, J. P. A.; KOGIKA, M. M. Tratado de Medicina Interna de Cães e Gatos. Rio de Janeiro: Roca, 2015. Cap. 185, p. 1666-1676.

CUNHA, E. L. A. Avaliação do tratamento com benznidazol, itraconazol e sua associação na fase aguda da doença de Chagas experimental no modelo cão. Ouro Preto: UFOP, 2017. 71p. Dissertação (Mestrado em Ciências Biológicas), Universidade Federal de Ouro Preto, 2017.

CUNNINGHAM, S. M. The Cardiovascular System in Animals. MSD Veterinary Manual. Disponível em: <https://www.msdvetmanual.com/circulatory-system/cardiovascularsystem-introduction/the-cardiovascular-system-in-animals\#v4499107> . Acesso em: 5 mai. 2019.

DANMAIGORO, A.; SELVARAJAH, G. T.; NOOR, M. H.; et al. Toxicity and safety evaluation of doxorubicin-loaded cockleshell-derived calcium carbonate nanoparticle in dogs. Advances in Pharmacological Sciences, v. 2018, p. 1-20, 2018. doi:

https://doi.org/10.1155/2018/4848602

DEFRANCESCO, T. C.; HAUCK, M. L. What's new in doxorubicin-induced cardiotoxicity. In: AMERICAN COLLEGE OF VETERINARY INTERNAL MEDICINE, 18, 2000, Seattle. PROCEEDINGS. Seattle: ACVIM, 2000. P. 121-122.

DILLON, A. R.; DELL'ITALIA, L. J.; TILLSON, M.; et al. Left ventricular remodeling in preclinical experimental mitral regurgitation of dogs. Journal of Veterinary Cardiology, v. 14, n. 1, p. 73-92, 2012. 
ELAMM, C.; FAIRWEATHER, D.; COOPER, L. T. Pathogenesis and Diagnosis of Myocarditis. Heart. British Cardiac Society, v. 98, n. 11, p. 835-840, 2012.

ESKOFIER, J.; WEFSTAEDT, P.; BEYERBACH, M.; et al. Quantification of left ventricular volumes and function in anesthetized beagles using real-time three-dimensional echocardiography: 4D-TomTec ${ }^{\mathrm{TM}}$ analysis versus 4D-AutLVQ ${ }^{\mathrm{TM}}$ analysis in comparison with cardiac magnetic resonance imaging. BMC Veterinary Research, v. 11, n. 260, p. 1-15, 2015.

FELDMAN, E. C.; NELSON, R. W. Hhypothyroidism. In: FELDMAN, E. C.; NELSON, R. W. Canine and feline endocrinilogy and reproduction. 3. ed. Philadelphia: WB Saunders, 2004. P. 86149.

FILIPPATOS, G.; ZANNAD, F. An introduction to acute heart failure syndromes: definition and classification. Heart Fail, v. 12, n. 2, p. 87-90, 2007.

FILIPPI, L. H. Arritmias. In: FILIPPI, L. H. O eletrocardiograma na medicina veterinária. São Paulo: Rocca, 2011. Cap. 12, p. 159-198.

FRUTUOSO, R. F. Doença degenerativa da valva mitral em cães. Vila Real: UTAD, 2015. 99p. Dissertação (Mestrado Integrado em Medicina Veterinária), Universidade de Trás-os-Montes e Alto Douro, 2015.

GUSTAFSON, D. L.; PAGE, R. L. Cancer Chemotherapy. In: WITHROW, S. J.; VAIL, D. M. Withrow \& MacEwen's - Small Animal Clinical Oncology. St. Louis: Elsevier Health Sciences, 2013. P. 157-171.

HÄGGSTRÖM, J.; KVART, C.; PEDERSEN, H. D. Acquired valvular hearth disease. In: ETTINGER, S. J.; FELDMAN, E. C. Textbook of Veterinary Internal Medicine. 6. ed. St Louis: Elsevier Saunders, 2010. P. 1022-1039.

HALLMAN, B. E.; HAUCK, M. L.; WILLIAMS, L. E.; et al. Incidence and risk factors associated with development of clinical cardiotoxicity in dogs receiving doxorubicin. Journal of Veterinary Internal Medicine, v. 33, n. 2, p. 783-791, 2019.

HOGAN, D. F.; GREEN, H. W. Dilated cardiomyopathy in dogs. In: BONAGURA, J. D.; TWEDT, D. C. Kirk's Current Veterinary Therapy. St. Louis: Saunders Elsevier, 2009. P. 792-797.

HOLLER, P. J.; WESS, G. Sphericity index and E-point-to-septal-separation (EPSS) to diagnose dilated cardiomyopathy in Doberman Pinschers. Journal of Veterinary Internal Medicine, $v$. 28, n. 1, p. 123-129, 2014.

HORI, Y.; TAIRA, H.; NAKAJIMA, Y.; et al. Inotropic effects of a single intravenous recommended dose of pimobendan in healthy dogs. Journal of Veterinary Medicine Science, v. 81, n. 1, p. 22-25, 2019. 
KAPLAN, J. L.; STERN, J. A.; FASCETTI, A. J.; et al. Taurine deficiency and dilated cardiomyopathy in golden retrievers fed commercial diets. PLoS ONE, v. 13, n. 12, p. 1-19, 2018.

KEENE, B. W.; ATKINS, C. E.; BONAGURA, J. D.; et al. ACVIM consensus guidelines for the diagnosis and treatment of myxomatous mitral valve disease in dogs. Journal of Veterinary Internal Medicine, v. 33, n. 3, p. 1127-1140, 2019.

MARTINS, P. S. A. Índice cardíaco vertebral em cães Dobermann: estudo através de um protocolo de rastreio de cardiomiopatia dilatada na raça. Lisboa: ULHT, 2017. 72p. Dissertação (Mestrado em Veterinária), Faculdade de Medicina Veterinária, Universidade Lusófona de Humanidades e Tecnologias, 2017.

MILLER, M. S.; et al. Electrocardiography. In: FOX, P. R.; SISSON, D.; MOISE, N. S. Small Animal Cardiology. 2. ed. Philadelphia: WB Saunders, 1999. Cap. 6.

MOONARMART, W.; BOSWOOD, A.; FUENTES, V. L.; et al. N-terminal pro B-type natriuretic peptide and left ventricular diameter independently predict mortality in dogs with mitral valve disease. Journal of Small Animal Practice, v. 51, n. 2, p. 84-96, 2010.

MORAES, H. A.; SCHWARTZ, D. S. Pathophysiology of Heart Failure. In: ETTINGER, S. J.; FELDMAN, E. C. Textbook of Veterinary Internal Medicine. Philadelphia: WB Saunders, 2005. P. 914-940.

MORAES, H. A. Fisiopatologia da insuficiência cardíaca e avaliação clínica da função cardíaca. In: ETTINGER, S. J; FELDMAN, E. C. Tratado de Medicina Interna Veterinária. 5. ed. Rio de Janeiro: Guanabara Koogan, 2014. Cap. 110, p. 732-752.

MURTA, D. J. M. C. Cardiomiopatia dilatada canina, a propósito de 13 casos clínicos. Lisboa: UTL, 2009. 132p. Dissertação (Mestrado em Veterinária), Faculdade de Medicina Veterinária, Universidade Técnica de Lisboa, 2009.

OCTAVIA, Y.; TOCCHETTI, C. G.; GABRIELSON, K. L.; et al. Doxorubicin-induced cardiomyopathy: From molecular mechanisms to therapeutic strategies. Journal of Molecular and Cellular Cardiology, v. 52, n. 6, p. 1213-1225, 2012.

O'GRADY, M. R.; O'SULLIVAN, M. L. Dilated cardiomyophaty: An update. The Veterinary Clinics of North America: Small Animal Practice, v. 34, n. 5, p. 1187-1207, 2004.

OLSEN, L. H.; HÄGGSTRÖM, J.; PEDERSEN, H. D. Acquired Valvular Heart Disease. In: ETTINGER, S. J.; FELDMAN, E. C. Textbook of Veterinary Internal Medicine. St Louis: Elsevier Saunders, 2010. P. 1299-1319.

PASLAWSKA, U.; NOSZCZYK-NOWAK, A.; NICPÓN, J. An assessment of left ventricular systolic function with the use of a continuous Doppler imaging in dogs with spontaneous mitral 
regurgitation. A preliminar study. Bulletin of the Veterinary Institute in Pulawy, v. 56, n. 3, p. 399-402, 2012.

PIVETA, R. B.; PAIVA, M. G.; ANDRADE, J. L. Diagnóstico por imagem da cardiotoxicidade. Revista da Sociedade de Cardiologia do Estado de São Paulo, v. 22, n. 3, p. 14-19, 2012.

RAHIMTOOLA, S. H. Digitalis therapy for patients in clinical heart failure. Circulation, v. 109, n. 24, p. 2942-2946, 2004.

RHODES, A.; EVANS, L. E.; ALHAZZANI, W.; et al. Surviving Sepsis Campaign: International Guidelines for Management of Sepsis and Septic Shock: 2016. Critical Care Medicine, v. 5, n. 3, p. 486-552, 2017.

SANTILLI, R. A.; PEREGO, M. Elettrocardiografia del cane e del gatto: genesi e interpretazione del ritmo cardiaco. Milano: Elsevier Masson, 2009. 276p.

SIESLACK, A. K.; DZIALLAS, P.; NOLTE, I.; et al. Comparative assessment of left ventricular function variables determined via cardiac computed tomography and cardiac magnetic resonance imaging in dogs. American Journal of Veterinary Research, v. 74, n. 7, p. 990-998, 2013.

SISSON, D.; O'GRADY, M. R.; CALVERT, C. A. Myocardial diseases of dog. In: SISSON, D.; O'GRADY, M. R.; CALVERT, C. A. Textbook of canine and feline cardiology. Principles and clinical practice. Philadelphia: Saunders Company, 1999. P. 581-620.

SISSON, D.; et al. Doença miocárdica primária no cão. In: ETTINGER, S. J.; FELDMAN, E. C. Tratado de Medicina Interna Veterinária. 5. ed. Rio de Janeiro: Guanabara Koogan, 2014. Cap. 116, p. 925-948.

SOUSA, M. G.; CARVALHO, E. R. Ecocardiografia. In: FELICIANO, M. A. R.; ASSIS, A. R.; VICENTE, W. R. R. Ultrassonografia em cães e gatos. São Paulo: MedVet, 2019. P. 365-438.

SOUZA, A. I.; JUNIOR, D. P.; SOUSA, M. G.; et al. Clinical and laboratorial features of naturally occurring Trypanosoma cruzi infection in dogs from Mato Grosso do Sul. Ciência Rural, v. 38, n. 5, p. 1351-1356, 2008.

SPINOSA, H. S; GÓRNIAK, S. L.; BERNARDI, M. M. Farmacologia Aplicada à Medicina Veterinária. 4. ed. Rio de Janeiro: Guanabara Koogan, 2006. 897p.

TILLEY, L. P.; SMITH, F. W.; OYAMA, M.; et al. Manual of Canine and Feline Cardiology. St. Louis: Elsevier Saunders, 2015. 472p.

VIDAL, B. L. Uso de inotrópicos positivos em cães e gatos. Brasília: UnB, 2016. 44p. Trabalho de Conclusão de Curso, Faculdade de Agronomia e Medicina Veterinária, Universidade de Brasília, 2016. 
VIÉRA, R. B. Toxicidade do cloridrato de doxorrubicina na dose de $120 \mathrm{mg} / \mathrm{m}^{2}$, em cadelas com tumor venéreo transmissível. Jaboticabal: UNESP, 2015. 66p. Dissertação (Mestrado em Veterinária), Faculdade de Ciências Agrárias e Veterinárias, Universidade Estadual Paulista/Jaboticabal, 2015.

VOLLMAR, A. C. Use of echocardiography in the diagnosis of dilated cardiomyopathy in Irish Wolfhounds. Journal of American Animal Hospital Association, v. 35, n. 4, p. 279-283, 1999.

VOORBIJ, A. M.; LEEGWATER, P. A.; BUIJTELS, J. J.; et al. Central hypothyroidism in miniature Schnauzers. Journal of Veterinary Internal Medicine, v. 30, n. 1, p. 85-91, 2016.

WARE, W. A. Myocardial disease of the dog. In: WARE, W. A. Cardiovascular Disease in Small Animal Medicine. Londres: Manson Publishing, 2011. P. 293-295.

WASSERSTROM, J. A.; AISTRUP, G. L. Digitalis: new actions for an old drug. American Journal of Physiology - Heart and Circulatory Physiology, v. 289, n. 5, p. 1-13, 2005.

WESS, G.; SCHULZE, A.; BUTZ, V.; et al. Prevalence of dilated cardiomyopathy in Doberman Pinschers in various age groups. Journal of Veterinary Internal Medicine, v. 24, n. 3, p. 533538, 2010.

WESS, G.; DOMENECH, O.; DUKES-MCEWAN, J.; et al. European Society of Veterinary Cardiology screening guidelines for dilated cardiomyopathy in Doberman Pinschers. Journal of Veterinary Cardiology, v. 19, n. 5, p. 405-415, 2017.

WOLF, M.; LUCINA, S. B.; BRÜLER, B. C.; et al. Assessment of longitudinal systolic function using tissue motion anular displacement in healthy dogs. Journal of Veterinary Cardiology, v. 20, n. 3, p. 175-185, 2018.

XUE, M.; CHEN, X.; GUO. Z.; et al. L-Carnitine attenuates cardiac dysfunction by ischemic insults through Akt signaling pathway. Toxicological Sciences, v. 160, n. 2, p. 341-350, 2017.

Autor para correspondência: Frederico Aécio Carvalho Soares. Faculdade de Veterinária, Centro Universitário Ritter dos Reis - UniRitter, Campus Fapa, Av. Manoel Elias, 2001, Passo das Pedras, CEP 91240-261, Porto Alegre (RS). fredaecio@gmail.com 